Revue de recherche interdisciplinaire sur le genre et la sexualité

35 | 2018

Striges en tous genres

\title{
Striges en tous genres : parions sur l'avenir !
}

\section{David Paternotte et Valérie Piette}

\section{OpenEdition \\ Journals}

Édition électronique

URL : https://journals.openedition.org/sextant/360

DOI : $10.4000 /$ sextant.360

ISSN : 2795-8736

Éditeur

Éditions de l'Université de Bruxelles

\section{Édition imprimée}

Date de publication : 1 décembre 2018

Pagination : 7-10

ISBN : 978-2-8004-1636-6

ISSN : $1370-267 X$

\section{Référence électronique}

David Paternotte et Valérie Piette, «Striges en tous genres : parions sur l'avenir ! », Sextant [En ligne] 35 | 2018, mis en ligne le 01 novembre 2021, consulté le 08 décembre 2021. URL : http:// journals.openedition.org/sextant/360; DOI : https://doi.org/10.4000/sextant.360

\section{(9) $(1)(0$}

La revue Sextant est mise à disposition selon les termes de la Licence Creative Commons Attribution Pas d'Utilisation Commerciale - Partage dans les Mêmes Conditions 4.0 International. 


\title{
Striges en tous genres : parions sur l'avenir !
}

\author{
David Paternotte et Valérie Piette ${ }^{1}$
}

En septembre 2015, un nouvel acteur est apparu sur la scène des études de genre : Striges ${ }^{2}$. Cette Structure de recherche interdisciplinaire sur le genre, l'égalité et la sexualité constitue une des équipes fondatrices de la Maison des Sciences humaines de l'ULB, née au même moment pour favoriser l'entreprise de recherches interdisciplinaires sur des objets précis (le genre, la ville, les mondes arabes et musulmans, l'Asie du Sud-Est et les Amériques). En échappant aux divisions facultaires parfois pesantes dans la vie de l'université, Striges permet aux architectes de collaborer avec des historien.ne.s et des juristes, aux psychologues de travailler avec des linguistes ou des anthropologues, aux sociologues de connaître des économistes et des philosophes. Cette structure, qui ne cesse de grandir, regroupe aujourd'hui une centaine d'enseignant.e.s et de chercheur.e.s issu.e.s de six facultés (Architecture, Droit et criminologie, Lettres, traduction et communication, Philosophie et sciences sociales, Psychologie et sciences de l'éducation, Solvay Brussels School of Economics and Management). Ses activités s'articulent autour de huit axes thématiques, qui constituent les principaux domaines d'expertise de ses membres : Religion et laïcité, Justice, égalité et non-discrimination, Espace(s), culture et diversité, Formation, travail et emploi, Corps, recherche et éducation, Construction épistémologique et dimensions éthiques et politiques. En outre, un séminaire mensuel - les Midis de Striges - permet de faire connaître les travaux menés au sein de l'équipe.

${ }^{1}$ Nous souhaitons remercier, pour leur travail sur ce numéro, Caroline Sägesser, qui coordonne notamment le projet ARC Sex\&Pil « Une spécificité belge ? Révolution sexuelle et (dé)pilarisation de 1960 à 2000 », et Natacha Chetcuti-Osorovitz, qui fut pendant un peu moins d'un an chercheure post-doctorante à Striges.

${ }^{2}$ http://msh.ulb.ac.be/equipes/striges/. 
$\mathrm{Si}$ cette structure est récente, elle s'appuie sur l'action de pionnières telles qu'Eliane Gubin, Bérengère Marques-Pereira, Danièle Meulders ou Eliane VogelPolsky, qui sont pour la plupart membres de Striges, ainsi que sur des expériences formelles et informelles plus anciennes comme la revue Sextant, fondée en 1993 par l'historienne Eliane Gubin ${ }^{3}$. Striges s'articule aussi aux initiatives facultaires comme la Chaire Suzanne Tassier-Charlier ${ }^{4}$ ou l'Atelier Genre(s) et Sexualité(s) de l'Institut de Sociologie. Cette structure ne se substitue donc pas aux initiatives facultaires existantes mais s'appuie sur celles-ci pour créer une coupole à l'échelle de l'Université. Elle est née de l'envie, surgie au cours du temps, de travailler ensemble et de construire collectivement quelque chose de durable. Striges souhaite en effet créer plus de synergies entre des collègues souvent isolé.e.s au sein de leur faculté, voire esseulé.e.s dans leur institut ou leur département, et poser ainsi les bases d'une institutionnalisation des études sur le genre et la sexualité à l'université. D'autant que cette institutionnalisation laisse souvent à désirer en Belgique, en comparaison avec la situation dans les pays voisins ${ }^{5}$.

Cette nouvelle structure permet de fédérer des énergies qui restaient trop souvent isolées et de réunir des collègues au-delà des frontières disciplinaires et facultaires. Des chercheur.e.s de droit, de psychologie et de sociologie ont ainsi collaboré à la réalisation d'un état des lieux de l'adoption en Fédération Wallonie-Bruxelles à la demande de cette dernière, tandis que des architectes, des linguistes et des historiennes préparent une exposition sur le porno en lien avec des associations de la société civile. En octobre 2017, Striges a aussi organisé le colloque « Troubles féministes dans l'islam et le judaïsme » sur une idée de Natacha Chetcuti. Ce projet, porté par l'équipe de la MSH de l'ULB et qui donnera lieu à un numéro thématique de Sextant, a réuni plusieurs équipes et centres de l'UlB et s'est construit à travers une relation étroite avec le Musée juif de Belgique. Enfin, les différentes équipes de la MSH organisent régulièrement des activités communes, notamment à l'occasion de la venue de professeur.e.s invité.e.s.

Striges s'inscrit enfin dans l'engagement ancien de l'ULB en faveur du développement d'études sur les femmes puis sur le genre et de soutien aux droits des femmes et des minorités sexuelles. Aujourd'hui, cette structure constitue le troisième pôle d'un triangle que nous espérons vertueux, dont il incarne le volet recherche. Il complète ainsi la politique de genre mise en place par Monique Tavernier à l'initiative du ministre Jean-Claude Marcourt et du recteur Didier Viviers, ainsi que le volet enseignement qui se développe progressivement. Le volet politique de genre ${ }^{6}$ se marque avant tout par la mise en place de structures de vigilance et de coordination à l'échelle de toute l'Université, sous la direction d'un vice-recteur à la politique

${ }^{3}$ http://msh.ulb.ac.be/equipes/striges/?sub=sextant.

${ }^{4} \mathrm{http}: / / \mathrm{msh} . u l b . a c . b e /$ equipes/striges/?sub=chaire-tassier.

${ }^{5}$ Groupe de TRAVAIL DU MASTER INTERUNIVERSITAIRE EN ÉTUDES DE GENRE, « Un master interuniversitaire en études de genre en Fédération Wallonie-Bruxelles : Coup d'œil dans le rétro, premier bilan et perspectives d'avenir ", Chronique féministe, 119, 2017, p. 45-48. Lire aussi S. BRACKE, «The Unbearable Lightness of 'Gender and Diversity' », Digest, 1/1, 2014, p. 41-50.

${ }^{6}$ http://www.ulb.ac.be/ulb/presentation/genre.html. 
de genre, aujourd'hui Laurent Licata, et d'une conseillère à la politique de genre, Danièle Meulders puis Valérie Piette. Cette politique porte une attention particulière à la carrière des femmes, une volonté qui se traduit notamment par la mesure Cascade une première en Fédération Wallonie-Bruxelles voire en Europe - qui garantit, parmi les promotions au rang suivant, un pourcentage d'hommes et de femmes similaire à celui du rang précédent. De nouvelles questions sont en train d'émerger, telles que le harcèlement sexuel, qui a fait l'objet d'une campagne conjointe avec l'Université de Genève en 2017, et la reconnaissance administrative des personnes trans. Le volet enseignement, longtemps constitué de cours isolés dans différentes facultés, est lui aussi en train de s'étoffer. D'une part, par une augmentation progressive du nombre de cours sur les questions de genre dans les différentes disciplines des sciences humaines. D'autre part, par la constitution de programmes entiers sur la question. Depuis quelques années, le master en sciences du travail offre une finalité genre et inégalités. En outre, le premier master ${ }^{7}$ de spécialisation en études de genre en Belgique francophone a vu le jour en septembre 2017. Issu des réflexions développées il y a quelques années par l'association Sophia, il rassemble les six universités de la Fédération Wallonie-Bruxelles et constitue le pendant du master Gender \& Diversiteit mis en place du côté flamand.

L'écrivaine britannique Virginia Woolf soulignait il y a presque un siècle que, pour pouvoir poursuivre son œuvre littéraire, une femme a besoin d'une rente et d'une chambre à soi. Si le financement des études de genre - bien que précaire - s'améliore dans notre pays, Striges s'efforce aujourd'hui d'offrir cet espace indispensable à la création. Attachée aux valeurs de notre Université, cette structure souhaite aussi poursuivre une tradition de mission à la société et jouer le rôle d'interface entre l'université, les pouvoirs publics et la société civile. En référence à son origine mythologique antique, Striges pousse un cri strident lorsqu'apparaissent des discriminations basées sur le sexe, le genre ou la sexualité.

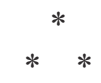

Consacrer un numéro de Sextant au travail réalisé dans cette nouvelle structure au lieu d'explorer une thématique particulière peut surprendre. Ce choix découle de la volonté de faire connaître la vivacité et la diversité du travail mené à Striges et ce numéro balaie par conséquent un large éventail de questions et de disciplines. En leur donnant une opportunité de publier leur travail, il montre aussi le rôle central des doctorant.e.s et des postdoctorant.e.s au sein de l'équipe. Ce rôle est d'ailleurs confirmé par l'organisation annuelle d'une journée doctorale et le lancement d'une école doctorale d'été francophone, le BRULAU, en collaboration avec le Centre d'Etudes genre de l'Université de Lausanne. Enfin, par l'inclusion de plusieurs hommages à des collègues et personnalités récemment décédées, il inscrit le travail de Striges dans une lignée qui le précède et revendique un héritage institutionnel qui rend possible le travail actuel.

Ce numéro s'inscrit dans un moment charnière de la vie de la revue. Longtemps marquée du sceau de l'UlB, Sextant opère pour l'instant la mue qui lui permettra de

\footnotetext{
${ }^{7}$ https://www.mastergenre.be.
} 
devenir la revue en études de genre de l'ensemble de la Fédération Wallonie-Bruxelles. Cela se traduit notamment par l'invitation à rejoindre le comité de rédaction adressée à Mylène Botbol-Baum (Université catholique de Louvain), Claire Gavray (Université de Liège), Nathalie Grandjean (Université de Namur) et Charlotte Pezeril (Université Saint-Louis). La revue s'apprête aussi à basculer dans la modernité numérique: après la numérisation de tous les anciens numéros dans la digithèque de l'ULB, elle entrera prochainement sur un portail de revues francophones. Enfin, la revue connaît une nouvelle direction. Si Vanessa Gemis continue à assurer le secrétariat de la revue, ce sont désormais Amandine Lauro et Cécile Vanderpelen qui manieront le gouvernail et le sextant. 